\title{
Optimizing provision of extracorporeal life support during the COVID-19 pandemic: practical considerations for Canadian jurisdictions
}

\author{
Ken Kuljit S. Parhar MD MSc, Laurance Lequier MD, Jaime Blackwood MD, Danny J. Zuege MD MSc, \\ Gurmeet Singh MD MSc
}

Cite as: CMAJ 2020 April 6;192:E372-4. doi: 10.1503/cmaj.200448; early-released March 26, 2020

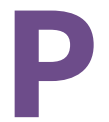

atients infected with the novel coronavirus, severe acute respiratory syndrome coronavirus 2 (SARS-CoV-2), may be asymptomatic or develop coronavirus disease 2019 (COVID-19), which is characterized by lower respiratory tract infection and inflammation. ${ }^{1}$ About $14 \%$ of people with COVID-19 develop severe disease, which may include acute respiratory distress syndrome. ${ }^{2}$ World Health Organization guidelines suggest that patients with COVID-19 with refractory hypoxemia despite lung protective ventilation be considered for extracorporeal life support (ECLS), ${ }^{3}$ an invasive cardiopulmonary support technology that uses an extracorporeal circuit for oxygen and carbon dioxide exchange. ${ }^{4}$ Extracorporeal life support is rescue therapy for patients who are not stabilized by other means of life support and who would not otherwise survive. It requires substantial resources, which are often limited even under nonpandemic circumstances. In this article, we discuss the utility of ECLS for viral pneumonia and describe the approach in Alberta, Canada, to optimal use of these resources during a pandemic, as our approach may be useful to other jurisdictions.

Use of ECLS has increased substantially over the past decade. This was catalyzed by 2 events: the CESAR (Conventional Ventilatory Support Versus Extracorporeal Membrane Oxygenation for Severe Adult Respiratory Failure) trial, a randomized controlled trial that showed a survival benefit for patients with respiratory failure referred to an ECLS centre, ${ }^{5}$ and the novel influenza A (H1N1) viral pandemic. The benefit of ECLS was demonstrated in several countries during the H1N1 pandemic. The experience of the Australian and New Zealand Intensive Care Society showed a greater than $70 \%$ survival of patients with H1N1-related acute respiratory distress syndrome who received ECLS. ${ }^{6} \mathrm{~A}$ study in the United Kingdom that compared patients with H1N1-related acute respiratory distress syndrome who received ECLS suggested a mortality benefit over propensity score-matched patients who did not. ${ }^{7}$ No mortality benefit was reported by a French research group; ${ }^{8}$ however, methodological differences and heterogeneity in unmatched patients in the study likely accounted for the discrepant results.

\section{KEY POINTS}

- COVID-19 pandemic preparation requires a multidisciplinary, stakeholder-guided approach, given that about $14 \%$ of people with COVID-19 develop severe disease, which may include acute respiratory distress syndrome.

- World Health Organization guidelines suggest that patients with COVID-19 with refractory hypoxemia despite lung protective ventilation be considered for extracorporeal life support (ECLS), which is a scarce resource that may require rationing in a pandemic situation.

- An accurate and easily understood strategy to communicate ECLS capacity is essential; in Alberta, we have developed a redyellow-green dashboard to indicate capacity and readiness.

- For large geographic areas, as in many of Canada's provinces and territories, clearly delineating the referral process for each feeder centre is important, and adult and pediatric ECLS centres may need to support one another.

- In the case of an overwhelming demand for critical care resources, ECLS services would need to be suspended in order to redirect all available critical care resources according to need.

The current COVID-19 pandemic is the third recent coronavirus outbreak of global importance, after severe acute respiratory syndrome (SARS) and Middle East respiratory syndrome (MERS). Extracorporeal life support was little used during the SARS outbreak. Small case series of patients with MERS showed an association between use of ECLS and improved survival, ${ }^{9}$ from which it is reasonable to presume that ECLS may provide survival benefits for selected patients with COVID-19-related severe acute respiratory distress syndrome. To date, the rapid pace of the COVID-19 pandemic has allowed for limited observational data and little time to conduct more rigorous efficacy studies. Large multicentre observational studies, such as the ECMO-CARD (Extracorporeal Membrane Oxygenation for 2019 Novel Coronavirus Acute Respiratory Disease) study, are underway, collecting real-time data to inform COVID-related ECLS practice. 
During pandemics, surges in patients requiring critical care resources are anticipated and can swiftly overwhelm a health care system. ${ }^{10}$ Public health efforts to slow person-to-person transmission could reduce the impact on critical care capacity. ${ }^{11}$ However, any infection or quarantining of health care providers will also strain critical care resources, including ECLS capacity. Triage decision-making and resource distribution are best centralized to ensure equity and justice in resource allocation during periods of high demand and strained supply of critical care resources.

An accurate and easily understood strategy to communicate ECLS capacity is essential. Capacity depends upon the number of patients possibly supportable with ECLS at any given time, as well as the number of patients who could be cannulated for ECLS, based on available disposable supplies. In Alberta, we developed a simple red-yellow-green dashboard, reflecting 3 readiness states (Appendix 1, supplemental Figure 1, available at www.cmaj.ca/lookup/suppl/doi:10.1503/ cmaj.200448/-/DC1). The green state is the number of runs performable and disposables available to continue operations, without affecting associated programs, such as critical care or cardiac surgery. The yellow state reflects that the current number of ECLS runs or available disposables are within a range in which rationing may be required. The red state indicates maximum ECLS capability, or that disposable capacity has reached critical levels. Operational impact on cardiac surgery and critical care are substantial at this stage. Throughout these states, individual ECLS programs should have robust strategies to support multiple circuits with varying degrees of staffing of ECLS specialists and perfusionists.

In Alberta, collaboration between adult and pediatric ECLS programs will facilitate sharing of equipment, disposable supplies and human resources. Further, bulk purchasing of disposables allows allocation among programs to maintain sufficient stock and meet clinical demand, provided supplies remain available.

To ensure fairness and efficiency, referral criteria for ECLS should be transparently outlined in advance. In Alberta, a subgroup of the provincial Critical Care Strategic Clinical Network's COVID-19 Pandemic Planning Group (Appendix 1, supplemental Table 1) has proposed ECLS referral based on a principled approach. Early assessment of each case by an ECLS consultant, who may have additional management suggestions before consideration for transfer to an ECLS centre or cannulation, can assist optimization of conventional medical therapy. We believe that having an ECLS consultant on call 24 hours per day, 7 days per week, should be standard of care. Patients with advanced age, high burden of medical comorbidities and poor likelihood of recovering sufficient lung function to promote independent recovery may be deemed unsuitable ECLS candidates. This is because it is unlikely that ECLS will offer additive therapeutic benefit beyond conventional management. Patients who have received mechanical ventilation for more than 7 days are unlikely to recover native lung function, even with rescue ECLS. ${ }^{4}$ Timing of referral from non-ECLS centres should consider the risks for transport of the critically ill, including weather limitations, transport time and patient stability, given a preference that cannulation take place at the ECLS centre. Making transport teams available for remote ECLS cannulation will likely not be possible when resources are substantially strained during a pandemic. Cases could be considered on an individual basis, however, depending on resource availability (Appendix 1, supplemental Table 2).

For large geographic areas, as in many of Canada's provinces and territories, clearly delineating the referral process for each feeder centre is important. In extraordinary circumstances, adult and pediatric ECLS programs may support one another through sharing of hardware, disposables, ECLS specialists and perfusionists, should one centre become overwhelmed.

Graded allocation of ECLS may be required as demands on resources change, with greater restriction required should resources become relatively scarce. Pandemic ethics usually apply utilitarian principles, with application, redirection or restriction of resources to those who can benefit most. ${ }^{12}$ Patients with the best perceived prognosis tend to be prioritized, necessitating more restrictive "entry" criteria and adaptation of usual "exit" criteria. Waning capacity of ECLS through attrition of staff or supplies or overutilization related to high demand should also be considered.

The province of Alberta has developed a 4-level system of triaging and safe operation of ECLS services (Appendix 1, supplemental Table 3), with level 1 indicating a state of sustainable operations, level 2 a state of increased strain on resources that will restrict an expanded group of patients from accessing ECLS, level 3 a high strain on resources requiring that ECLS be restricted to only those patients with the highest probability of survival, and level 4 an overwhelming demand for critical care resources, in which ECLS services would be suspended to redirect all available critical care resources to other critically ill patients. Decision-making to move through each of the 4 triaging stages is centralized to an emergency command centre that incorporates up-to-date information from all ECLS and critical care programs related to service demand and health care capacity.

In summary, COVID-19 pandemic preparation requires a multidisciplinary, stakeholder-guided approach. Rational deployment of ECLS ensures that this scarce and expensive, lifesaving technology is available to as many patients as possible who may benefit.

\section{References}

1. Zhu N, Zhang D, Wang W, et al. A novel coronavirus from patients with pneumonia in China, 2019. N Engl J Med 2020;382:727-33.

2. Wu Z, McGoogan JM. Characteristics of and important lessons from the coronavirus disease 2019 (COVID-19) outbreak in China: summary of a report of 72314 cases from the Chinese Center for Disease Control and Prevention. JAMA 2020 Feb. 24. [Epub ahead of print]. doi: 10.1001/jama.2020.2648.

3. Clinical management of severe acute respiratory infection when novel coronavirus ( $\mathrm{nCoV}$ ) infection is suspected [interim guidance]. Geneva: World Health Organization; 2020.

4. Parhar K, Vuylsteke A. What's new in ECMO: scoring the bad indications. Intensive Care Med 2014;40:1734-7

5. Peek GJ, Mugford M, Tiruvoipati R, et al. Efficacy and economic assessment of conventional ventilatory support versus extracorporeal membrane oxygenation for severe adult respiratory failure (CESAR): a multicentre randomised controlled trial. Lancet 2009;374:1351-63. 
6. Australia and New Zealand Extracorporeal Membrane Oxygenation Influenza (ANZ ECMO) Investigators; Davies A, Jones D, Bailey M, et al. Extracorporeal membrane oxygenation for 2009 influenza $A(H 1 N 1)$ acute respiratory distress syndrome. JAMA 2009;302:1888-95.

7. Noah MA, Peek GJ, Finney SJ, et al. Referral to an extracorporeal membrane oxygenation center and mortality among patients with severe 2009 influenza A(H1N1). JAMA 2011;306:1659-68.

8. Pham T, Combes A, Roze H, et al. Extracorporeal membrane oxygenation for pandemic influenza $\mathrm{A}(\mathrm{H} 1 \mathrm{~N} 1)$-induced acute respiratory distress syndrome: a cohort study and propensity-matched analysis. Am J Respir Crit Care Med 2013;187:276-85.
9. Alshahrani MS, Sindi A, Alshamsi F, et al. Extracorporeal membrane oxygenation for severe Middle East respiratory syndrome coronavirus. Ann Intensive Care 2018;8:3.

10. Grasselli G, Pesenti A, Cecconi M. Critical care utilization for the COVID-19 outbreak in Lombardy, Italy. JAMA 2020 Mar. 13 [Epub ahead of print]. doi:10.1001/ jama.2020.4031.

11. Murthy S, Gomersall CD, Fowler RA. Care for critically ill patients with COVID-19 JAMA 2020 Mar. 11. [Epub ahead of print]. doi: 10.1001/jama.2020.3633.

12. Kaposy C, Bandrauk N, Pullman D, et al. Adapting the Hamilton Health Sciences critical care pandemic triage protocol. Healthc Q 2010;13:60-3.
Competing interests: Gurmeet Singh reports receiving consulting fees from Edwards Lifesciences as a member of the advisory board. Ken Parhar is a member of the advisory board for Elsius Biomedical but has received no direct or indirect compensation. Dr. Parhar has received research grant funding from the Canadian Institutes of Health Research and the MSI Foundation. No other competing interests were declared.

This article was solicited and has not been peer reviewed.

Affiliations: Department of Critical Care Medicine (Parhar, Zuege), University of Calgary and Alberta Health Services, Calgary, Alta.; Department of Pediatrics - Division of Critical Care (Lequier), University of Alberta and Alberta Health Services, Edmonton, Alta.; Department of Pediatrics - Division of Critical Care (Blackwood), University of Calgary and Alberta Health Services; Critical Care Strategic Clinical Network (Zuege), Alberta Health Services, Calgary, Alta.; Departments of Critical Care Medicine and Surgery, Division of Cardiac Surgery (Singh), University of Alberta and Alberta Health Services, Edmonton, Alta.
Contributors: Ken Kuljit Parhar and Gurmeet Singh contributed to the conception and design of the work and writing of the original draft. All of the authors contributed to drafting the manuscript and revising it critically for important intellectual content. All of the authors gave final approval of the version to be published and agreed to be accountable for all aspects of the work.

Acknowledgements: The authors acknowledge ECLS coordinators Nicole Dubyk, Don Granoski, Steve Menzies and Tanya Spence for their review of the manuscript and suggestions. The authors wish to thank the chair of the committee, Kristin Robertson, and the membership of the Alberta Health Services Provincial Critical Care COVID-19 ECLS subgroup for their contributions in the development of the pandemic planning documentation. Most importantly, the authors recognize the skill and dedication of ECLS specialists, perfusionists and all other health care professionals who comprise the vast team required to deliver life-saving technology to the critically ill.

Correspondence to: Ken Kuljit Singh Parhar, ken.parhar@albertahealthservices.ca 